\title{
Does It Make Sense? And Why? A Pilot Study for Sense Making and Explanation
}

\author{
Cunxiang Wang ${ }^{1,4}$, Shuailong Liang ${ }^{2}$, Yue Zhang1, Xiaonan $\mathrm{Li}^{3}$ and Tian Gao \\ ${ }^{1}$ School of Engineering, Westlake University, China \\ ${ }^{2}$ Singapore University of Technology and Design, Singapore \\ ${ }^{3}$ School of Computer Science and Technology, Xidian University, China \\ ${ }^{4}$ College of Computer Science and Technology, Zhejiang University, China \\ wangcunxiang@westlake.edu.cn, yue.zhang@wias.org.cn \\ shuailong_liang@mymail.sutd.edu.sg \\ lixiaonan_xdu@outlook.com, gaotian@zju.edu.cn
}

\begin{abstract}
Introducing common sense to natural language understanding systems has received increasing research attention. It remains a fundamental question on how to evaluate whether a system has a sense making capability. Existing benchmarks measures commonsense knowledge indirectly and without explanation. In this paper, we release a benchmark to directly test whether a system can differentiate natural language statements that make sense from those that do not make sense. In addition, a system is asked to identify the most crucial reason why a statement does not make sense. We evaluate models trained over large-scale language modeling tasks as well as human performance, showing that there are different challenges for system sense making.
\end{abstract}

\section{Introduction}

Natural Language Understanding (NLU) has received increasing research attention in recent years. With language models trained on large corpora (Peters et al., 2018; Devlin et al., 2018), algorithms show better performance than humans on some benchmarks (Group, 2017; Devlin et al., 2018). Compared to humans, however, most endto-end trained systems are rather weak on common sense. For example, it is straightforward for a human to understand that someone can put a turkey into a fridge but he can never put an elephant into a fridge with basic commonsense reasoning, but it can be non-trivial for a system to tell the difference. Arguably, commonsense reasoning should be a central capability in a practical NLU system (Davis, 2017); it is, therefore, important to be able to evaluate how well a model can do for sense making.

Existing datasets test common sense indirectly through tasks that require extra knowledge, such

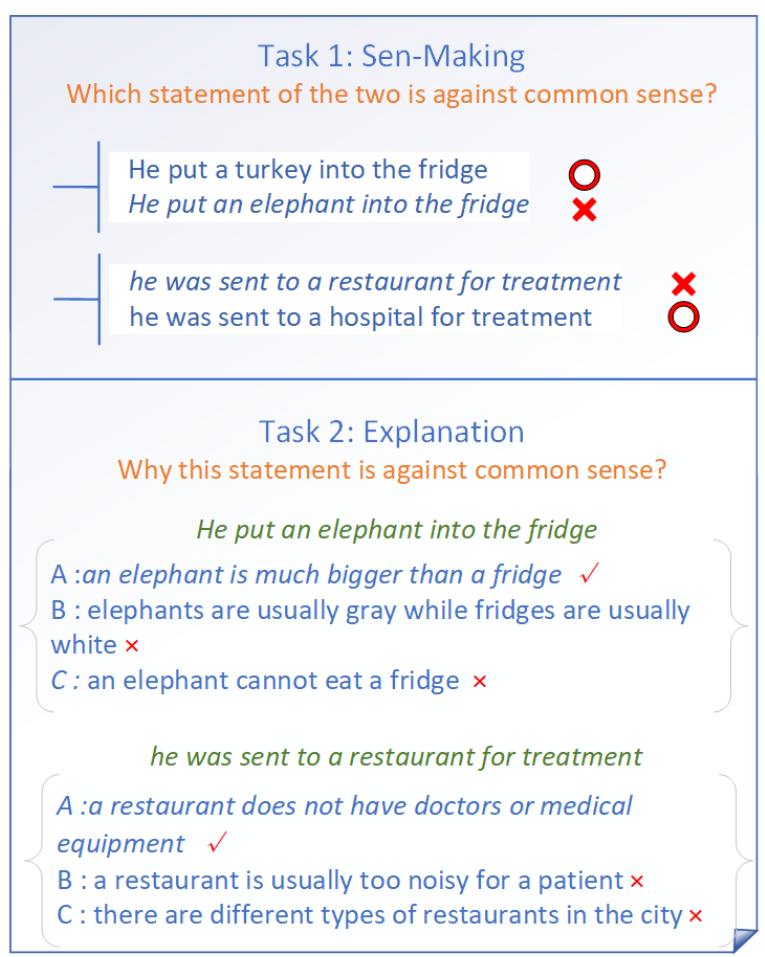

Figure 1: Samples of our dataset

as co-reference resolution (Levesque et al., 2012; Morgenstern and Ortiz, 2015), subsequent event prediction (Roemmele et al., 2011; Zhang et al., 2017; Zellers et al., 2018), or reading comprehension (Mostafazadeh et al., 2016; Ostermann et al., 2018b). They verify whether a system is equipped with common sense by testing whether it can give a correct answer where the input does not contain such knowledge. However, there are two limitations to such benchmarks. First, they do not give a direct metric to quantitatively measure sense making capability. Second, they do not explicitly identify the key factors required in a sense making process.

We address these issues by creating a testset for direct sense making. As shown in Figure 1, the 
benchmark consists of two subtasks. The first task is to choose from two natural language statements with similar wordings which one makes sense and which one does not make sense; The second task is to find the key reason why a given statement does not make sense. Version 1.0 of our dataset, which will be released with this paper, contains 2021 instances for each subtask, manually labeled by 7 annotators. Human performance on the benchmark is $99.1 \%$ for the Sen-Making task and $97.3 \%$ for the Explanation task.

In the Sen-Making task, we use statement pair classification rather than labelling each statement 'true' or 'false' in the absolute sense because it is easy to cite a counterexample for any single 'true' or 'false' statement. For example, 'toy elephant' for 'he put an elephant into fridge'. But it is apparent that 'he put a turkey into fridge' is more reasonable than 'he put an elephant into fridge'.

In this pilot study, we evaluate contextualized representations trained over large-scale language modeling tasks on our benchmark. Results show that there is still a large gap behind human performance despite that the models are trained over 100 million natural language sentences. Detailed examination shows that inference remains a challenge for such systems. To our knowledge, our dataset has the most direct decision-making process in commonsense reasoning and is the first one asking reasons behind the decision making process.

Note that there has been dataset which focus on non-linguistic world knowledge plausibility (Wang et al., 2018) or only limited attributes or actions of physical knowledge like verbphysics (Forbes and Choi, 2017). They are related to our dataset but serve robotic research mainly. Our dataset is the first benchmark for direct linguistic sense making and explanation. We hope this benchmark can promote commonsense reasoning by the NLP community, and further applied on other applications such as machine translation and dialogue. Besides, we also expect that this work could be instructive on enhancing interpretability on commonsense reasoning research and other NLP tasks and on combining explanation with language generation. Our dataset is released at https://github.com/wangcunxiang/SenMaking-and-Explanation.

\section{Related Work}

There has been datasets which focus on nonlinguistic world knowledge validation (Wang et al., 2018) or only limited attributes or actions of world knowledge (Forbes and Choi, 2017)

A widely used and important task is the Winograd Schema Challenge (WSC) (Levesque et al., 2012; Morgenstern and Ortiz, 2015) that needs more commonsense knowledge. For example, "The trophy would not fit in the brown suitcase because it was too big (small). What was too big (small)?" "Answer 0: the trophy", "Answer 1: the suitcase". However, WSC estimates common sense indirectly and it does not consider explanation on why one option is true while the other is wrong.

Choice of Plausible Alternatives (COPA) (Roemmele et al., 2011) puts emphasis on events and consequences. Each question of COPA asks to find the suitable cause or result of the premise from two given alternatives. All premises and alternatives are simple sentences. For example, "Premise: The man broke his toe. What was the CAUSE of this?" "1: He got a hole in his sock.", "2: He dropped a hammer on his foot.". Several subsequent datasets are inspired by COPA. The JHU Ordinal Common-sense Inference (JOCI) (Zhang et al., 2017) aims to label the plausibility from 5 (very likely) to 1 (impossible) of human response after a certain situation. Situations with Adversarial Generations (SWAG) (Zellers et al., 2018) requests to choose the most likely-to-happen alternative after a specific situation. Those datasets put emphasis on the presituations and/or the after-situations of certain situations, but not on the reasons why they occur or lead.

Some datasets are inspired by reading comprehension, providing some textual materials and questions, asking to find suitable answers from the provided materials. The Story Cloze Test and ROCStories Corpora (Mostafazadeh et al., 2016; Sharma et al., 2018) require to figure out the right ending from two candidate sentences after a foursentence story. For a narrative text, MCScript (Ostermann et al., 2018a) give various types of questions and pairs of answer candidates for each question. Most questions require knowledge beyond the facts mentioned in the text. Compared to those reading comprehension tasks, our benchmark encourages people to use any external resources they 
want as long as they will help in the task.

Some other datasets are evolved from QA problems and care more about factual commonsense knowledge. SQUABU (Davis, 2016) provides a small hand-constructed test of commonsense and scientific questions. CommonsenseQA (Talmor et al., 2018) asks crowd workers to create questions from ConceptNet (Speer et al., 2017), which is a large knowledge graph of commonsense knowledge, where each question discriminates its answer candidates between three target concepts that all share the same relationship to a single source drawn from ConceptNet OpenBookQA (Mihaylov et al., 2018) provides questions and answer candidates, as well as thousands of diverse facts about elementary level science that are related to the questions. The AI2 Reasoning Challenge (ARC) (Clark et al., 2018) gives thousands of questions with different knowledge types, as well as a relevant 14M-sentence corpus, mixed with science facts and other narrative sentences. Those questions are not easy to answer without specializing certain knowledge while our questions are easy for both adults and children.

In contrast, to our work, the tasks above do not directly estimate common sense or ask the logical reasons behind the correct answers and questions.

In recent years, some large-scale commonsense inference knowledge resources have been released, which may be helpful in commonsense reasoning tasks. Atomic (Sap et al., 2018) presents a huge everyday commonsense reasoning knowledge graph, which has nine if-then relations with variables, including causes, effects, and so on. Event2Mind (Rashkin et al., 2018) proposes a new corpus and task, aiming to find out mentioned/unmentioned people's intents and reactions under various daily circumstances. These datasets are not directly useful for our benchmark since they focus only on a small domain. ConceptNet is a prestigious knowledge graph that has been upgraded over a long time (Liu and Singh, 2004; Havasi et al., 2007; Speer and Havasi, 2013; Speer et al., 2017). ConceptNet constructs triples using labeled edges as relations and various words and/or phrases as entities. It also has the sentences describing the corresponding triples. Thus we consider using ConceptNet knowledge for finetuning our models.

\section{Testset}

Task. Formally, each instance in our dataset is composed of 5 sentences: $\left\{s_{1}, s_{2}, r_{1}, r_{2}, r_{3}\right\} . s_{1}$ and $s_{2}$ are two similar sentences which in the same syntactic structure and differ by only few words, but only one of them makes sense while the other does not. They are used on our first subtask called Sen-Making, which requires the model to identify which one is valid. For the invalid sentence, we have three optional reasons $r_{1}, r_{2}$ and $r_{3}$ to explain why the sentence is invalid. Our subtask 2, named Explanation, requires that the only one correct reason be identified from two other confusing ones. We use the accuracy score to evaluate both subtasks.

Data. For version 1.0 of our dataset, we have created 2,021 samples. Task 1 has 2,021 againstcommon-sense sentences, 2,021 correct sentences; Task 2 has 2,021 true reasons and 4,042 confusing reasons. We plan to release a dataset with more tasks and samples in the future.

Annotation guidelines. When writing samples, annotators were asked to follow several principles. First, try to avoid complex knowledge and focus on daily common sense, and should make the questions as understandable as possible. Every literate person is able to give the right answers. Second, the confusing reasons should better contain more important words like entities and activities in the against-common-sense statements, for example, the confusing reasons of "he put an elephant into the fridge" should better contain both "elephant" and "fridge". Third, we want the confusing reasons to be related to the statements and correct reasons and not deviate from the problem context, otherwise, it may be easily captured by BERT (Talmor et al., 2018), which models the sentence contexts explicitly. Next, the three option reasons should be only related to the incorrect sentence rather the correct sentence. Because we want further studies to be able to estimate against-common-sense statements without those correct statements. Furthermore, the confusing sentences should be correct themselves. Otherwise, the models may simply ignore the incorrect options without considering the casual relations between them. This worry was raised from the fact that models can achieve high performance in the ROC Story Cloze Task when only looking at the alternative endings ignoring the stories (Schwartz et al., 2017). Last, we control the length of each 


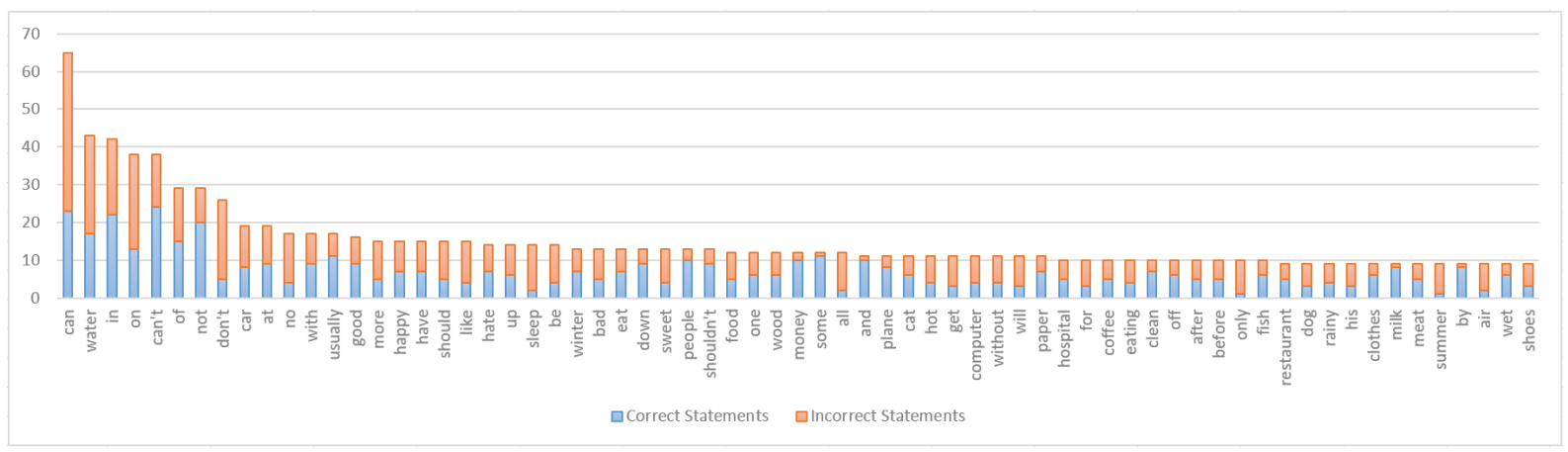

Figure 2: Number of 'Different Words'

sentence, making the incorrect statement nearly as long as the correct statement, and the right reason neither too long nor too short among the three reasons. However, those principles are all soft restrictions; some samples are still good ones even without obeying those principles.

Annotation process. We ask data annotators to write samples by themselves. Then other researchers (the first and second author of this paper) will examine those samples case by case. We also provide them with external sources to stimulate inspiration, such as the raw English sentences of ConceptNet5.5 (Speer et al., 2017), but those inspirational sentences should not be used directly. For example, "he was sent to a (restaurant)/(hospital) for treatment after a car crash" were inspired by the two sentences "restaurants provide food" and "hospitals provide medical care" However, those corpus may have incorrect and one-sided knowledge, we do not use those sentences. Besides, we also let them get inspiration from existing commonsense reasoning questions like WSC (Levesque et al., 2012; Morgenstern and Ortiz, 2015), COPA (Roemmele et al., 2011) and SQUABU (Davis, 2016).

Cautions when using the data. 1 . Researchers are encouraged to use what they deemed appropriate for the tasks to train their model, and use our testset for evaluation. 2. Please do not use the three optional reasons when performing SenMaking task, or the task will be artificially easy.

\subsection{Corpus Analysis}

The average length of two statements in the SenMaking task are both 8.26, exactly the same. The average length of true reasons is 7.63 , slightly smaller than the confusing reasons' average length, which is 7.77 .

We analyse the different words between correct statements and incorrect statements, like in
"He put (an elephant)/(a turkey) into the fridge", 'an elephant' is 'different word' in incorrect statements and 'a turkey' is 'different word' in correct statements. From 'different words', we can get an overview about how correct statements and incorrect statements differ from each other.

We select the words which appear more than 9 times in the 'different words' and remove stopwords like 'a', 'an' and 'the' to draw Figure 2. blue lines are the words appear in correct statements but do not appear in incorrect statements, while orange lines are opposite. We find that incorrect statements have much the same negative different words compared with correct statements. The incorrect statements have 53 'don't' or 'can't' or 'not' or 'no' as different words, while the correct statements have 55. That can illustrate that the corpus do not use negative words to construct incorrect statements or correct statements.

\section{Experiments}

We choose state-of-the-art language models trained over large texts as our baselines, assuming that common sense knowledge are encoded over texts. For the sense making task, we calculate perplexities of both statements, choosing the one with lower scores as the correct one. For explanation, we first concatenate the statement with the each reason and then use the three concatenated sentences to calculate perplexities. For example, we concatenate "he put an elephant into the fridge" with its optional reasons to be "he put an elephant into the fridge" is against common sense because an elephant cannot eat a fridge".

We also conduct human evaluation on our dataset. Each sample is answered by at least three testees. If more than half testees do one sample wrong (either Sen-Making or Explanation), we will rewrite or abolish the sample. Otherwise, we 


\begin{tabular}{|c|c|c|}
\hline Model & Sen-Making & Explanation \\
\hline Random & $50.0 \%$ & $33.3 \%$ \\
\hline ELMo & $69.4 \%$ & $33.4 \%$ \\
\hline BERT & $70.1 \%$ & $45.6 \%$ \\
\hline fine-tuned ELMo & $74.1 \%$ & $34.6 \%$ \\
\hline Human Performance & $99.1 \%$ & $97.8 \%$ \\
\hline
\end{tabular}

Table 1: Experimental Results

will keep it and record it in results.

\subsection{Results \& Analysis}

As shown in Table 1, ELMo and BERT have a significant advantage over random results in SenMaking. We conjure that both of them have the ability to judge whether a sentence is with or against common sense. For Sen-Making, ELMo does better than BERT; However, BERT beats ELMo in Explanation. ELMo does poorly in Explanation, much the same as a random guess, which shows that ELMo cannot handle the casual relationship between the incorrect statements and the reasons. In contrast, BERT is significantly better than random guess in Explanation. This can be attributed to BERT's training on Next Sentence Prediction (Devlin et al., 2018), which can assist to handle the logic relationship between two sentences.

Fine-tuned ELMo has an obvious improvement in Sen-Making and a non-obvious improvement in Explanation, probably because introducing knowledge will help models to identify common sense but cannot help them in inference. However, fine-tuning makes BERT perform the same in Sen-Making and even worse in Explanation. It is likely because the original BERT models trained on BookCorpus (Zhu et al., 2015) and English Wikipedia contain sufficient common knowledge and the fine-tune operation may be useless or even makes the models be specific in the finetuning corpora; Besides, fine-tuning may corrupt the structure formed by Next Sentence Prediction. How to overcome the weaknesses of fine-tuning operation may be an interesting topic in representation learning.

The method of fine-tuning can be viewed as a way to make use of human labelled knowledge base for common sense, however, a thorough investigation along this direction takes significant research effort, which is beyond the scope of this paper. It is also important to note that human labelled commonsense knowledge bases are in- evitably limited by the scope. Therefore, gleaning knowledge from large raw text is still a more promising direction.

Note also that unlike reading comprehension tasks (Rajpurkar et al., 2016), where machines can surpass human performance by careful finetuning, it remains a big challenge for systems to reach human performance, which is near $100 \%$ for sense making. When the human testee is asked to look at the mistakes that they make, we find that most human errors are due to reduced concentration rather than conceptual issues.

\subsection{Case Study}

We find an interesting case illustrating that finetuning operation can help model identify common sense but cannot help much in inference. The example is "New York is located in the northeastern part of USA" vs "the USA is located in the northeastern part of New York". The original ELMo is incorrect in both Sen-Making and Explanation. After fine-tuning with a corpora which contains that "New York is a city" and "the USA is a country", the ELMo can figure out the incorrect sentence but still cannot pick out the correct reason.

We find that LM-based techniques cannot handle commonsense Sen-Making problems which need inference, such as the case "I' $m$ too full for lunch, so I'm going (for a walk)/(to eat more)." and its explanation, no matter fine-tuned or not. This is likely because it remains difficult for LSTM language models for making multi-step inference.

\section{Conclusion}

We created a benchmark for directly evaluating whether a system has the capability of sense making and explanation, evaluating models trained over the large raw text as well as a common sense database on the test set. Results show that sense making remains a technical challenge for such models, whereas inference is a key factor that is missing. 


\section{Acknowledgement}

We thank the anonymous reviewers for constructive suggestions, and the non-author data annotators Run'ge Yan, Chenyan Du, Zinqun Zhou and Qikui Feng. The work is supported by NSFC grant number 61572245 . Yue Zhang is the corresponding author.

\section{References}

Peter Clark, Isaac Cowhey, Oren Etzioni, Tushar Khot, Ashish Sabharwal, Carissa Schoenick, and Oyvind Tafjord. 2018. Think you have solved question answering? try arc, the AI2 reasoning challenge. CoRR, abs/1803.05457.

Ernest Davis. 2016. How to write science questions that are easy for people and hard for computers. AI Magazine, 37:13-22.

Ernest Davis. 2017. Logical formalizations of commonsense reasoning: a survey. Journal of Artificial Intelligence Research, 59:651-723.

Jacob Devlin, Ming-Wei Chang, Kenton Lee, and Kristina Toutanova. 2018. Bert: Pre-training of deep bidirectional transformers for language understanding. arXiv preprint arXiv:1810.04805.

Maxwell Forbes and Yejin Choi. 2017. Verb physics: Relative physical knowledge of actions and objects. In $A C L$.

Natural Language Computing Group. 2017. R-net: Machine reading comprehension with self-matching networks.

Catherine Havasi, Robert Speer, and Jason Alonso. 2007. Conceptnet 3: a flexible, multilingual semantic network for common sense knowledge. In Recent advances in natural language processing, pages 27 29. Citeseer.

Hector J. Levesque, Ernest Davis, and Leora Morgenstern. 2012. The winograd schema challenge. In Proceedings of the Thirteenth International Conference on Principles of Knowledge Representation and Reasoning, KR'12, pages 552-561. AAAI Press.

Hugo Liu and Push Singh. 2004. Conceptnet-a practical commonsense reasoning tool-kit. BT technology journal, 22(4):211-226.

Todor Mihaylov, Peter Clark, Tushar Khot, and Ashish Sabharwal. 2018. Can a suit of armor conduct electricity? a new dataset for open book question answering. In Proceedings of the 2018 Conference on Empirical Methods in Natural Language Processing, pages 2381-2391. Association for Computational Linguistics.
Leora Morgenstern and Charles L. Ortiz. 2015. The winograd schema challenge: Evaluating progress in commonsense reasoning. In Proceedings of the Twenty-Ninth AAAI Conference on Artificial Intelligence, AAAI'15, pages 4024-4025. AAAI Press.

Nasrin Mostafazadeh, Nathanael Chambers, Xiaodong He, Devi Parikh, Dhruv Batra, Lucy Vanderwende, Pushmeet Kohli, and James F. Allen. 2016. A corpus and cloze evaluation for deeper understanding of commonsense stories. In HLT-NAACL.

Simon Ostermann, Ashutosh Modi, Michael Roth, Stefan Thater, and Manfred Pinkal. 2018a. Mcscript: A novel dataset for assessing machine comprehension using script knowledge. In Proceedings of the Eleventh International Conference on Language Resources and Evaluation (LREC-2018). European Language Resource Association.

Simon Ostermann, Michael Roth, Ashutosh Modi, Stefan Thater, and Manfred Pinkal. 2018b. Semeval2018 task 11: Machine comprehension using commonsense knowledge. In Proceedings of The 12th International Workshop on Semantic Evaluation, pages 747-757. Association for Computational Linguistics.

Matthew E. Peters, Mark Neumann, Mohit Iyyer, Matt Gardner, Christopher Clark, Kenton Lee, and Luke Zettlemoyer. 2018. Deep contextualized word representations. In Proc. of NAACL.

Pranav Rajpurkar, Jian Zhang, Konstantin Lopyrev, and Percy Liang. 2016. Squad: 100,000+ questions for machine comprehension of text. In Proceedings of the 2016 Conference on Empirical Methods in Natural Language Processing, pages 2383-2392. Association for Computational Linguistics.

Hannah Rashkin, Maarten Sap, Emily Allaway, Noah A. Smith, and Yejin Choi. 2018. Event2mind: Commonsense inference on events, intents, and reactions. In Proceedings of the 56th Annual Meeting of the Association for Computational Linguistics (Volume 1: Long Papers), pages 463-473. Association for Computational Linguistics.

Melissa Roemmele, Cosmin Adrian Bejan, and Andrew S. Gordon. 2011. Choice of Plausible Alternatives: An Evaluation of Commonsense Causal Reasoning. In AAAI Spring Symposium on Logical Formalizations of Commonsense Reasoning, Stanford University.

Maarten Sap, Ronan LeBras, Emily Allaway, Chandra Bhagavatula, Nicholas Lourie, Hannah Rashkin, Brendan Roof, Noah A. Smith, and Yejin Choi. 2018. ATOMIC: an atlas of machine commonsense for if-then reasoning. CoRR, abs/1811.00146.

Roy Schwartz, Maarten Sap, Ioannis Konstas, Leila Zilles, Yejin Choi, and Noah A. Smith. 2017. The effect of different writing tasks on linguistic style: A case study of the roc story cloze task. In Proceedings of the 21st Conference on Computational 
Natural Language Learning (CoNLL 2017), pages 15-25. Association for Computational Linguistics.

Rishi Sharma, James Allen, Omid Bakhshandeh, and Nasrin Mostafazadeh. 2018. Tackling the story ending biases in the story cloze test. In Proceedings of the 56th Annual Meeting of the Association for Computational Linguistics (Volume 2: Short Papers), pages 752-757. Association for Computational Linguistics.

Robert Speer and Catherine Havasi. 2013. Conceptnet 5: A large semantic network for relational knowledge. In The People's Web Meets NLP, pages 161176. Springer.

Robyn Speer, Joshua Chin, and Catherine Havasi. 2017. Conceptnet 5.5: An open multilingual graph of general knowledge.

Alon Talmor, Jonathan Herzig, Nicholas Lourie, and Jonathan Berant. 2018. Commonsenseqa: A question answering challenge targeting commonsense knowledge. CoRR, abs/1811.00937.

Su Wang, Greg Durrett, and Katrin Erk. 2018. Modeling semantic plausibility by injecting world knowledge. arXiv preprint arXiv:1804.00619.

Rowan Zellers, Yonatan Bisk, Roy Schwartz, and Yejin Choi. 2018. Swag: A large-scale adversarial dataset for grounded commonsense inference. In Proceedings of the 2018 Conference on Empirical Methods in Natural Language Processing (EMNLP).

Sheng Zhang, Rachel Rudinger, Kevin Duh, and Benjamin Van Durme. 2017. Ordinal common-sense inference. Transactions of the Association for Computational Linguistics, 5:379-395.

Yukun Zhu, Ryan Kiros, Rich Zemel, Ruslan Salakhutdinov, Raquel Urtasun, Antonio Torralba, and Sanja Fidler. 2015. Aligning books and movies: Towards story-like visual explanations by watching movies and reading books. In Proceedings of the IEEE international conference on computer vision, pages 19-27. 